\title{
On the Splitting of the Dual Goldie Torsion Theory
}

\author{
Christian Lomp
}

\begin{abstract}
The splitting of the Goldie (or singular) torsion theory has been extensively studied. Here we determine an appropriate dual Goldie torsion theory, discuss its splitting and answer in the negative a question proposed by Özcan and Harmancı as to whether the splitting of the dual Goldie torsion theory implies the ring to be quasi-Frobenius.
\end{abstract}

\section{Introduction}

Let $R$ be an associative ring with unit and $M$ a left $R$-module. A submodule $N$ of $M$ is called essential in $M$ (denoted by $N \unlhd M$ ) if $N \cap L \neq 0$ holds for every non-zero submodule $L$ of $M$. A left $R$-module $M$ is called singular if there are left $R$-modules $L$ and $K$ such that $K$ is an essential submodule of $L$ and $M \simeq L / K$. We denote the class of singular left $R$-modules by $R$-Sing and the sum of all singular submodules of a module $M$ by $Z\left({ }_{R} M\right):=\operatorname{trace}(R$-Sing, $M)$, where the trace of a class of modules into a module is defined by $\operatorname{trace}(\mathcal{X}, M):=$ $\sum\{\operatorname{Im}(f): f \in \operatorname{Hom}(X, M), X \in \mathcal{X}\}$. The lattice-theoretical dual of the notion of essential submodules are small submodules $N$ of $M$ (denoted by $N \ll M$ ) that have the property that $N+L \neq M$ holds for every proper submodule $L$ of $M$. Dual to a singular module, a left $R$-module $M$ is called small if there are left $R$-modules $K$ and $L$ such that $K$ is a small submodule of $L$ and $M \simeq K$. A definition of small and singular objects of abelian categories was given by B.Pareigis (see $[\mathbf{P}]$ ). It is easily verified that $M$ is a small left $R$-module if and only if it is a small submodule of its injective hull $E(M)$ (see for example [ $\mathbf{Z}$, Theorem 1]). We denote the class of small left $R$-modules by $R$-Small and denote the sum of all small submodules of a module $M$ by $Z^{*}\left({ }_{R} M\right):=\operatorname{trace}(R$-Small, $M)$. The class of small modules is closed under submodules, homomorphic images and finite direct sums. Note that a simple left $R$-module is either singular or projective and either small or injective. Small and singular modules occur in decomposition theorems of modules over quasi Frobenius rings (QF-rings).

1991 Mathematics Subject Classification. Primary 13C12, 16G10.

This note was done with the support of "DAAD Doktorandenstipendium HSP III" while the author was visiting the Department of Mathematical Science at UW-Milwaukee 1997/98. He would like to thank the department for their support and hospitality. Moreover he would like to express his thanks to Ayşe Ç. Özcan for supplying him with her paper and her thesis and to Mark L. Teply for his valuable comment and for Proposition 4.10. 
Theorem 1.1 (Rayar [R/R2, Theorem 7, Corollary 11], Oshiro [0]). Let $R$ be a ring. The following are equivalent:

(a) $R$ is a QF-ring;

(b) every left $R$-module is a direct sum of a projective and a small module;

(c) every left $R$-module is a direct sum of an injective and a singular module;

(d) $\operatorname{Soc}\left({ }_{R} R\right) \subseteq \operatorname{Soc}\left(R_{R}\right)$ holds and

(i) every left $R$-module is a direct sum of an injective and a small module or

(ii) every right $R$-module is a direct sum of a projective and a singular module.

Recently Özcan and Harmanc $\iota$ proved in $\mathbf{O H}$ that modules over QF-rings have a direct sum decomposition into two "orthogonal" classes of modules. In Section 2 we will realize those two module classes as a torsion and torsion free class of a hereditary torsion theory - the dual Goldie torsion theory - and state some basic properties. Those rings whose dual Goldie torsion theory is trivial or improper will be studied in Section 3. Especially those rings $R$ that are small $R$-submodules of their injective hull will be discussed. In Section 4 we will determine when the dual Goldie torsion theory splits. In particular we will characterize semilocal rings whose dual Goldie torsion theory is splitting as those that cogenerate all injective simple left $R$-modules. Hence semilocal left Kasch rings have this property. We will give a list of classes of rings whose dual Goldie torsion theory is splitting, but that are far from being QF. This answers in the negative a question of Özcan and Harmanc $\imath$ as to whether the splitting of the dual Goldie torsion theory implies the ring to be QF (see $\mathbf{O H}$, pp 325]). For all unexplained ring- and module-theoretical notations we refer to [W91]; for all unexplained torsion-theoretical notations we refer to [G].

\section{Dual Goldie Torsion Theory}

Let $\mathcal{X}$ be a class of left $R$-modules closed under ismorphisms and submodules. Define the following classes:

$$
\begin{aligned}
& \mathbb{F}(\mathcal{X}):=\{M \in R \text {-Mod } \mid \forall X \in \mathcal{X}: \operatorname{Hom}(X, M)=0\} \\
& \mathbb{T}(\mathcal{X}):=\{N \in R \text {-Mod } \mid \forall M \in \mathbb{F}(\mathcal{X}): \operatorname{Hom}(N, M)=0\}
\end{aligned}
$$

Then $(\mathbb{T}(\mathcal{X}), \mathbb{F}(\mathcal{X}))$ is a hereditary torsion theory (see $\mathbf{B K N}$, II1.3]), the smallest hereditary torsion theory such that all $\mathcal{X}$-modules are torsion. The torsion radical is $\tau_{\mathcal{X}}(M)=\operatorname{trace}(\mathbb{T}(\mathcal{X}), M)$ and $\operatorname{trace}(\mathcal{X}, M) \unlhd \tau_{\mathcal{X}}(M)$ holds. This kind of torsion theory has also been extensively studied by Harmanc $\imath$ and Smith, where the corresponding torsion radical was denoted by $\underline{H}^{*}$ (see in $[\mathbf{H S}$ ).

The following are easily checked:

$$
\begin{aligned}
& \mathbb{T}(\mathcal{X})=\{N \in R \text {-Mod } \mid \forall U \subset V \subseteq N: \operatorname{trace}(\mathcal{X}, V / U) \neq 0\} \\
& \mathbb{F}(\mathcal{X})=\{M \in R \text {-Mod } \mid \operatorname{trace}(\mathcal{X}, M)=0\}
\end{aligned}
$$

The Goldie torsion theory is defined as: $\tau_{G}=(\mathbb{T}(R$-Sing $), \mathbb{F}(R$-Sing $))$. V.S. Ramamurthi defined a dual Goldie torsion theory as $\tau_{c G}=(\mathbb{T}(R$-Small $), \mathbb{F}(R$-Small $))$ and studied some of its properties in $[\mathbb{R}]$. We have $Z^{*}(M)=M \cap \operatorname{Rad}(E(M)) \unlhd$ $\tau_{c G}(M)$ for every left $R$-module $M$.

The class of singular modules is closed under arbitrary direct sums, but does not have to be closed under extensions in general. In case of a nonsigular ring $R$-Sing $=\mathbb{T}(R$-Sing $)$. The class of small modules is, in general, neither closed under 
arbitrary direct sums nor under extensions. A necessary and sufficient condition for $R$-Small to be closed under direct sums is that every injective left $R$-module has a small radical (see $\mathbf{R 8 2}$, Lemma 9]).

Related to $\tau_{c G}$ are two classes of modules introduced by Özcan and Harmancı in $\mathbf{O H}$ :

$$
\begin{aligned}
\underline{X} & :=\left\{M \in R-\operatorname{Mod} \mid Z^{*}(M)=0\right\} \\
\underline{X}^{*} & :=\left\{M \in R \text {-Mod } \mid \forall U \subset V \subseteq M: Z^{*}(V / U) \neq 0\right\}
\end{aligned}
$$

From (2.1) we see that $\tau_{c G}=\left(\underline{X}^{*}, \underline{X}\right)$. Özcan and Harmanc $\imath$ showed that over a QF-ring every left $R$-module is a direct sum of an $\underline{X}$-module and an $\underline{X}^{*}$-module. In other words they showed that $\tau_{c G}$ splits over QF-rings. They raised the question, "Is a ring $R$ whose dual Goldie torsion theory $\tau_{c G}$ is splitting a QF ring ? (see $\mathbf{O H}$, pp 325]). It is not difficult to see that this question has a negative answer, and we will discuss the splitting of $\tau_{c G}$ in the sequel.

A different approach to defining a dual Goldie torsion theory was proposed by A.I. Generalov in $\mathbf{G e}$. Let $\mathcal{X}$ be a class of left $R$-modules closed under ismorphisms and submodules. Define the following classes:

$$
\begin{aligned}
& \mathbb{T}^{\perp}(\mathcal{X}):=\{M \in R \text {-Mod } \mid \forall X \in \mathcal{X}: \operatorname{Hom}(M, X)=0\} \\
& \mathbb{F}^{\perp}(\mathcal{X}):=\left\{N \in R \text {-Mod } \mid \forall M \in \mathbb{T}^{\perp}(\mathcal{X}): \operatorname{Hom}(M, N)=0\right\}
\end{aligned}
$$

Then $\left(\mathbb{T}^{\perp}(\mathcal{X}), \mathbb{F}^{\perp}(\mathcal{X})\right)$ is a torsion theory, not necessarily hereditary. Denote the reject of $\mathcal{X}$ in $M$ by $\operatorname{reject}(M, \mathcal{X}):=\bigcap\{\operatorname{ker} g: g \in \operatorname{Hom}(M, X), X \in \mathcal{X}\}$. Then we see as above:

$$
\begin{aligned}
& \mathbb{T}^{\perp}(\mathcal{X})=\{M \in R \text {-Mod } \mid \operatorname{reject}(M, \mathcal{X})=M\} \\
& \mathbb{F}^{\perp}(\mathcal{X})=\{N \in R \text {-Mod } \mid \forall 0 \subset U \subseteq M: \operatorname{reject}(U, \mathcal{X}) \neq U\}
\end{aligned}
$$

The torsion class $\mathbb{T}^{\perp}(R$-Small $)$ has the property that $M \in \mathbb{T}^{\perp}(R$-Small $)$ if and only if every factor module of $M$ is $\tau_{c G^{-}}$-torsion free. We denote this torsion theory by $\tau_{c G}{ }^{\perp}$, for if $\tau_{c G}{ }^{\perp}$ is hereditary, then it is the pseudo-complement of $\tau_{c G}$ in the lattice of hereditary torsion theories $R$-tors (see $[\mathbf{G}$, pp. 280]).

A characterization of $\tau_{c G}{ }^{\perp}$-torsion modules was given in $[\mathbf{G e}]$ and [ $[\mathbf{L o}]$ :

Proposition 2.1 (Lomp [Lo, 5.1], Generalov [Ge, Proposition 3]). Let $R$ be a ring and let $M$ be a left $R$-module. Then the following conditions are equivalent:

(a) $M$ is $\tau_{c G}{ }^{\perp}$-torsion;

(b) $\forall K \in R$-Mod and $0 \neq f: M \rightarrow K, \operatorname{Im}(f) \nless K$;

(c) $\forall K \in R$-Mod and $0 \neq f: M \rightarrow K, \operatorname{Im}(f)$ is coclosed in $K$, i.e. $\operatorname{Im}(f) / L \nless$ $K / L$ for all $L \subset \operatorname{Im}(f)$;

(d) for every factor module $L$ of $M$ and diagram in $R$-Mod

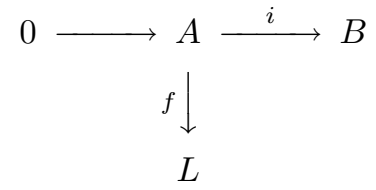

there exists a factor module $\pi: L \rightarrow \tilde{L}$ and a $g \in \operatorname{Hom}(B, \tilde{L})$ such that $i g=f \pi$ holds.

The following result is easily verified: 
LEMma 2.2. $\mathbb{F}(R$-Small $)=\mathbb{T}^{\perp}(R$-Small $)$ if and only if $\tau_{c G}$ is cohereditary and $\tau_{c G}^{\perp}$ is hereditary.

The torsion radical of $\tau_{c G}{ }^{\perp}$ can also be defined via an inductive construction of a preradical $\rho$ given by Generalov: for any module $M$ let $\sigma^{1}(M):=$ $\operatorname{reject}\left(M, R\right.$-Small), for any ordinal number $\alpha$ define $\sigma^{\alpha+1}(M):=\operatorname{reject}\left(\sigma^{\alpha}(M), R\right.$-Small $)$ and if $\alpha$ is a limit ordinal, let $\sigma^{\alpha}(M):=\bigcap_{\beta<\alpha} \sigma^{\beta}(M)$. Then there exists an ordinal number $\gamma$ such that $\sigma^{\gamma}(M)=\sigma^{\gamma+1}(M)$. Hence define a preradical by $\rho(M):=\sigma^{\gamma}(M)$. Generalov showed that $\rho$ is an idempotent preradical, that defines the torsion class $\mathbb{T}^{\perp}(R$-Small), and called it the dual Goldie torsion theory.

\section{V-Rings, Small Rings and Almost Small Rings}

All torsion theories are considered as torsion theories of left $R$-modules unless otherwise specified. We will use the torsion theoretical notion for $\xi$ to denote the trivial torsion theory (all non-zero modules are torsion free ) and $\chi$ to denote the improper torsion theory (all modules are torsion).

3.1. V-Rings. We first examine when $\tau_{c G}$ becomes trivial.

Proposition 3.1. Let $R$ be a ring. Then $R$ is a left $V$-ring if and only if $\tau_{c G}=\xi$ if and only if ${ }_{R} R$ is $\tau_{c G}{ }^{\perp}$-torsion.

Proof. Simple modules are either small or injective (see [R82]). If $\tau_{c G}=\xi$, then all simple left $R$-modules are injective and hence $R$ is a left $V$-ring. On the other hand, if $R$ is a left $V$-ring, then $\operatorname{Rad}(M)=0$ for all left $R$-modules $M$. Hence there are no non-zero small left $R$-modules and $\tau_{c G}=\xi$. Obviously $\tau_{c G}=\xi \Leftrightarrow \tau_{c G}{ }^{\perp}=\chi \Leftrightarrow{ }_{R} R$ is $\tau_{c G}{ }^{\perp}$-torsion.

If $\tau_{c G}=\xi$, then $\tau_{c G}$ is trivially splitting; e.g. for a direct product of fields $\tau_{c G}$ is splitting. The following example of a $\tau_{c G}$-torsion free $\operatorname{ring} R$ with $\tau_{c G} \neq \xi$ shows that $R$ being $\tau_{c G}$-torsion free is not sufficient for $R$ to be a $V$-ring.

EXAmple 3.2. The endomorphism ring $S$ of an infinite dimensional vector space $V_{k}$ over a field $k$ is a von Neumann regular left self-injective ring, but not a left $V$-ring (since ${ }_{S} V$ is a simple, non-injective $S$-module and hence small, see W91, 23.6]). As $S$ is left self-injective, we have Jac $(S)=Z^{*}\left({ }_{S} S\right)$. Moreover $\operatorname{Jac}(S)=0$, as $S$ is von Neumann regular. Thus $S$ is $\tau_{c G}$-torsion free, but $\tau_{c G} \neq \xi$.

3.2. Small Rings. We will now examine when $\tau_{c G}$ becomes improper. A ring $R$ is called left small if ${ }_{R} R$ is a small module; e.g. $\mathbb{Z}$ is a small ring as it is small in $\mathbb{Z} \mathbb{Q}$. A left small ring $R$ is $\tau_{c G}$-torsion as left $R$-module. Hence every left $R$-module is $\tau_{c G}$-torsion and we have $\tau_{c G}=\chi$.

Proposition 3.3 (Ramamurthi [ R, 3.3], Pareigis [ [P, Satz 4.8]). Let $R$ be a ring and let $E(R)$ be the injective hull of ${ }_{R} R$. Then the following conditions are equivalent:

(a) $R$ is a left small ring;

(b) $\operatorname{Rad}(M)=M$ for every injective left $R$-module $M$;

(c) $\operatorname{Rad}(E(R))=E(R)$.

Harada showed that a commutative ring is small in its classical quotient ring

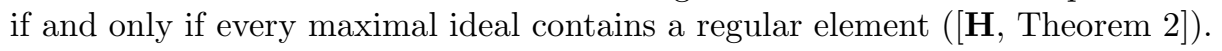


THEOREM 3.4. Let $R$ be a ring such that every left primitve ideal contains a regular element. Then $R$ is left small.

Proof. Assume $R$ is not small in its injective hull $E:=E(R)$. Then there exists a submodule $N \subseteq E$ such that $R+N=E$ as left $R$-modules. Hence $E / N$ is cyclic and has a maximal submodule $M / N$. The left annihilator $I:=l(E / M)$ of the left simple $R$-module $E / M$ contains a regular element $x$ by hypothesis. As $E$ is a divisible left $R$-module, we have $x(E / M)=0 \Rightarrow E=x E \subseteq M$, which is a contradiction. Hence $R$ has to be small in $E(R)$.

As a corollary we get the following known results:

Corollary 3.5. Proper integral domains (Pareigis, [ $\mathbf{P}$, Folgerung 5.3]), rings whose Jacobson radical contains a regular element (Ramamurthi, $\mathbf{R}$, Proposition 3.4]) and prime left Goldie rings that are not left primitive (Özcan, [Oz , 2.2.5]) are small rings.

Recall that a left $R$-module $M$ is torsionfree (in the usual sense) if $r m \neq 0$ holds for every $m \in M$ and $r \in R$. The following result shows that small rings bring some restrictions.

Proposition 3.6. Let $R$ be a left small ring, $E$ an injective left $R$-module and $\lambda$ the cardinality of an generating set of $E$. Then $\lambda \geq \aleph_{0}$ and $R^{(\lambda)}$ is not a small left $R$-module. Moreover the cardinality of any independent family of submodules of a finitely generated torsionfree left $R$-module $M$ is bounded by $\lambda$. If $\lambda=\aleph_{0}$, every finitely generated torsionfree left $R$-module has finite Goldie dimension.

Proof. First note that $E$ is generated by $R^{(\lambda)}$ and that an injective left $R$ module cannot be small. Therefore $\lambda$ has to be infinite and $R^{(\lambda)}$ cannot be a small module. If we have an independent family of cardinality $\lambda^{\prime}$ of cyclic submodules of a finitely generated torsionfree left $R$-module $M$, then $R^{\left(\lambda^{\prime}\right)}$ embeds into $M$. If $\lambda^{\prime} \geq \lambda$, then $R^{\left(\lambda^{\prime}\right)}$ is not small. Hence $M$ is not small, which contradicts the fact that $M$ is a small module as it is a homomorphic image of a finite direct sum of copies of the small module ${ }_{R} R$. Thus $\lambda^{\prime}<\lambda$.

REMARK 3.7. Small rings form a class of rings where small modules are not closed under arbitrary direct sums. Contrary to this class are left max rings. A ring $R$ is called a left $\max$ ring if every left $R$-module has a maximal submodule, or equivalently, if every left $R$-module has a small radical. By M.Rayar's result mentioned above, $R$-Small is closed under arbitrary direct sums over a max ring $R$. It is well-known that $R$ is left perfect if and only if $R$ is a semilocal left max ring. Hence we already encountered a huge class of rings, namely the small rings, whose dual Goldie torsion theory (trivially) splits, but that cannot be perfect (nor QF) answering in the negative a question of Özcan and Harmanc $\imath$ as to whether the splitting of $\tau_{c G}$ implies the ring to be QF. As an additional observation together with 3.4, we have that a left max domain has to be left primitive.

3.3. Almost Small Rings. Let us call a ring $R$ left almost small if $\tau_{c G}=\chi$. Obviously every left small ring is left almost small, and a ring $R$ is left almost small if and only if ${ }_{R} R$ is $\tau_{c G}$-torsion. So $\tau_{c G}$ trivially splits for left almost small rings.

Proposition 3.8. Any local ring is either a division ring or else left and right almost small. 
Proof. Let $R$ be a local ring. If $R / \operatorname{Jac}(R)$ is injective, then $R$ is a left $V$-ring. Hence $\operatorname{Jac}(R)=0$, which implies $R$ is a division ring. If $R$ is not a division ring, then $R / \operatorname{Jac}(R)$ must be a small left (or right) $R$-module. Thus $R$ is $\tau_{c G}$-torsion, as $\operatorname{Jac}(R)$ is small and $\tau_{c G}$-torsion modules are closed under extensions. Hence $R$ is left and right almost small.

\section{The splitting of the dual Goldie torsion theory.}

There are many example of rings where the dual Goldie torsion theory splits. M.Rayar proved in [R87, Proposition 1] that a direct product of a family of proper integral domains is a small ring. Hence any product of integral domains is a direct product of a small and a $V$-ring; thus $\tau_{c G}$ splits. Take, for instance, $R=\mathbb{Z}^{\mathbb{N}} \times \mathbb{Q}^{\mathbb{N}}$.

We will need the next technical lemma to investigate torsion theories of finite products of rings.

LEMMA 4.1. Let $R_{1}, \ldots, R_{n}$ be rings and denote by $R:=R_{1} \times \cdots \times R_{n}$ their direct product with componentwise multiplication. For all $i$, let $\mathcal{X}_{i} \subseteq R_{i}-$ Mod be classes of left $R_{i}$-modules closed under submodules and isomorphisms and set $\mathcal{X}:=\bigoplus_{i=1}^{n} \mathcal{X}_{i}$. Then $\mathbb{F}(\mathcal{X})=\bigoplus_{i=1}^{n} \mathbb{F}\left(\mathcal{X}_{i}\right)$ and $\mathbb{T}(\mathcal{X})=\bigoplus_{i=1}^{n} \mathbb{T}\left(\mathcal{X}_{i}\right)$ (where the $\mathbb{F}\left(\mathcal{X}_{i}\right)$, resp. $\mathbb{T}\left(\mathcal{X}_{i}\right)$, are formed in $R_{i}-$ Mod $)$.

Proof. Note that the unit of $R$ is $1=\left(1_{R_{1}}, \ldots, 1_{R_{n}}\right)$ and that $R=\bigoplus_{i=1}^{n} R_{i}$ as a left $R$-module. Hence for all $M \in R-\operatorname{Mod}$ we have $M=\sum_{i=1}^{n} M_{i}$ with $M_{i}:=$ $R_{i} M$. Every left $R_{i}$-module $M$ becomes a left $R$-module by $\left(r_{1}, \ldots, r_{n}\right) \cdot m:=r_{i} m$. Note that $R_{i} \cdot M_{j}=0$ if $i \neq j$. Therefore every $M \in R-\operatorname{Mod}$ can be decomposed as $M=\bigoplus_{i=1}^{n} M_{i}$. Let $M \in R_{i}-\operatorname{Mod}$ and $N \in R-\operatorname{Mod}$ such that $N_{i}=0$; then $\operatorname{Hom}_{R}(N, M)=0$. Now let $N, M \in R-\operatorname{Mod}$ :

$$
\operatorname{Hom}(M, N) \simeq \bigoplus_{i=1}^{n} \bigoplus_{j=1}^{n} \operatorname{Hom}\left(M_{i}, N_{j}\right)=\bigoplus_{i=1}^{n} \operatorname{Hom}\left(M_{i}, N_{i}\right)
$$

Hence $\operatorname{trace}(\mathcal{X}, M)=\bigoplus_{i=1}^{n} \operatorname{trace}\left(\mathcal{X}_{i}, M_{i}\right)$ holds, which implies $\mathbb{F}(\mathcal{X})=\bigoplus_{i=1}^{n} \mathbb{F}\left(\mathcal{X}_{i}\right)$ and $\mathbb{T}(\mathcal{X})=\bigoplus_{i=1}^{n} \mathbb{T}\left(\mathcal{X}_{i}\right)$.

Thus as a direct consequence we get the following corollary (note that $R$-Small $=$ $\bigoplus_{i=1}^{n} R_{i}$-Small holds). Let us denote by $R_{i} \tau$ a torsion theory in $R_{i}$-tors.

Corollary 4.2. Let $R=R_{1} \times \cdots \times R_{n}$. Then ${ }_{R} \tau_{c G}=\left(R_{1} \tau_{c G}\right) \times \cdots \times\left(R_{n} \tau_{c G}\right)$.

As a consequence of Corollary 4.2 and the facts that every commutative semiperfect ring is a direct product of local rings and every local ring is either a division ring or almost small by Proposition 3.8, we observe:

COROLlary 4.3. Any commutative semiperfect ring is a direct product of an almost small ring and a semisimple ring.

A torsion theory $\tau$ is called cohereditary if the class of $\tau$-torsionfree modules is closed under homomorphic images. Example 3.2 shows that $\tau_{c G}$ does not have to be cohereditary. With the help of the above Corollary 4.2 we can determine when $\tau_{c G}$ splits for rings with cohereditary dual Goldie torsion theory:

THEOREM 4.4. Let $R$ be a ring. Then the following conditions are equivalent:

(a) $\tau_{c G}$ is splitting and cohereditary;

(b) $R \simeq T \times S$ with $T$ a left almost small and $S$ a left $V$-ring; 
Proof. $(a) \Rightarrow(b)$ Let ${ }_{R} R={ }_{R} T \oplus_{R} S$ with ${ }_{R} T=\tau_{c G}\left({ }_{R} R\right)$ and ${ }_{R} S$ being $\tau_{c G}$-torsion free. We have $\operatorname{Hom}(T, S)=0$, and thus $T S=0$ implies that $T$ is an ideal in $R$. As the class of $\tau_{c G}$-torsion free modules is closed under homorphic images, we have $\operatorname{Hom}(S, T)=0$ and thus $S T=0$. Hence $T$ and $S$ are ideals with $T \cap S=0$. Thus $R=T \times S$, and as $\tau_{c G}$ is cohereditary, all $\tau_{c G}$-torsion left $R$ modules are generated by ${ }_{R} T$ and all $\tau_{c G}$-torsion free left $R$-modules are generated by ${ }_{R} S$. Thus $T$ is left almost small and $S$ a left V-ring.

$(b) \Rightarrow(a)$ By Corollary 4.2 we have $(\mathbb{T}(R$-Small $), \mathbb{F}(R$-Small $))=(T-M o d, S-$ $M o d)$, and every left $R$-module $M$ can be decomposed into $M=T M \oplus S M$. Thus $\tau_{c G}$ is splitting. $\tau_{c G}$ is cohereditary since $\mathbb{F}(R$-Small $)=S$-Mod.

A module $M$ is called cosemisimple if $\operatorname{Rad}(M / K)=0$ for all $K \subset M$. All $\tau_{c G^{-}}$ torsion free modules are cosemisimple in case that $\tau_{c G}$ is cohereditary. In the next proposition we will characterize when $\tau_{c G}$ is cohereditary. Recall that a module $M$ is called $\tau$-injective with respect to a hereditary torsion theory $\tau$ if $M$ is injective with respect to all short exact sequences $0 \rightarrow L \rightarrow N \rightarrow K \rightarrow 0$ such that $K$ is $\tau$-torsion.

Proposition 4.5. Let $R$ be a ring. Then the following conditions are equivalent:

(a) $\tau_{c G}$ is cohereditary;

(b) all $\tau_{c G}$-torsion free left $R$-modules are $\tau_{c G}$-injective;

(c) $E(M) / M$ is $\tau_{c G}$-torsion free for all $\tau_{c G}$-torsion free left $R$-modules $M$.

If one of the above conditions hold, then all $\tau_{c G}$-torsion free left $R$-modules are cosemisimple.

Proof. $(a) \Rightarrow(c)$ is trivial; $(c) \Leftrightarrow(b)$ holds for any torsion theory (see $\mathbf{W 9 6}$ ). (b) $\Rightarrow(a)$ Since $\tau_{c G}$ is hereditary, every submodule $N$ of a $\tau_{c G}$-torsion free module $M$ with $M / N$ being $\tau_{c G}$-torsion satisfies $M \simeq N \oplus M / N$ and so $N=M$.

For semilocal rings $R$ the dual Goldie torsion theory becomes rather simple. Let $R$-Simp, and let $\mathcal{C}$, resp. $\mathcal{I}$, denote the class of simple, semisimple, resp. injective, left $R$-modules. Before we state a more general result about the splitting of $\tau_{c G}$. We note that the following equivalences hold:

$$
\mathbb{F}(R \text {-Small }) \subseteq \mathcal{C} \Leftrightarrow \mathbb{F}(R \text {-Small }) \subseteq \mathcal{I} \Leftrightarrow \mathbb{F}(R \text {-Small })=\mathcal{C} \cap \mathcal{I} \text {. }
$$

Moreover in this situation $\tau_{c G}$ is cohereditary and the dual Goldie torsion theory is nothing but the torsion theory cogenerated by the injective simple left $R$-modules; i.e. $\tau_{c G}=\chi\{R$-Simp $\cap \mathcal{I}\}$. We have this situation when $R / Z^{*}\left({ }_{R} R\right)$ is semisimple; e.g., $R$ is semilocal.

THEOREM 4.6. Let $R$ be a ring with $R / Z^{*}\left({ }_{R} R\right)$ semisimple. Then the following conditions are equivalent:

(a) $\tau_{c G}$ is splitting;

(b) $R \simeq T \times S$ with $T$ a left almost small and $S$ a semisimple ring;

(c) $\tau_{c G}$ is stable (i.e. the $\tau_{c G}$-torsion class is closed under essential extensions);

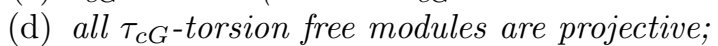

(e) all injective simple left $R$-modules are projective;

(f) $\tau_{G} \leq \tau_{c G}$ (i.e, $\tau_{c G}$ is a generalization of $\tau_{G}$ );

(g) all singular left $R$-modules are $\tau_{c G}$-torsion;

(h) $R$ cogenerates all injective simple left $R$-modules. 
Proof. If $R / Z^{*}\left({ }_{R} R\right)$ is semisimple, then all $\tau_{c G}$-torsion free modules are semisimple as $Z^{*}\left({ }_{R} R\right) M=0$. By the above we have $\mathbb{F}(R$-Small $)=\mathcal{C} \cap \mathcal{I}$; thus $\tau_{c G}$ is cohereditary. Hence Theorem 4.4 gives us $(a) \Leftrightarrow(b)$. Note that $(b) \Leftrightarrow(c)$ by $\mathbf{G}$, Prop. 5.10], $(c) \Leftrightarrow(d)$ by $[\mathbf{G}$, E5.10], and $(d) \Leftrightarrow(e)$ is obvious. To show $(d) \Rightarrow(f)$ let $M$ be $\tau_{c G}$-torsion free; then $Z(M)$ being projective implies $Z(M)=0$ and $M$ is non-singular. Hence all $\tau_{c G}$-torsion free modules are $\tau_{G}$-torsion free: $\tau_{G} \leq \tau_{c G}$. The implication $(f) \Rightarrow(g)$ is trivial. To prove $(g) \Rightarrow(e)$, note that an injective simple module cannot be small; therefore it cannot be singular by hypothesis and must be projective. Finally, $(e) \Leftrightarrow(h)$ is obvious.

REMARK 4.7. Semilocal rings satisfy the hypothesis of Theorem 4.6. A ring $R$ is called a left Kasch ring if it cogenerates all left simple $R$-modules. Of course any left cogenerator ring (e.g., left PF-ring or QF-ring) is a left Kasch ring, but the converse does not hold in general. (A ring is called left PF-ring if it is left self-injective and left Kasch.)

As a corollary of Theorem 4.6 we note:

Corollary 4.8. For any semilocal left Kasch ring, $\tau_{c G}$ splits.

Remark 4.9. A result by Faith and Menal [FM] states that a left Kasch ring with finite right uniform dimension is semilocal. Another result by Gómez Pardo and Yousif [GPY] states that a left Kasch, right CS ring is semiperfect. Hence for those rings $\tau_{c G}$ splits.

A sufficient and necessary condition for a semilocal ring to be left Kasch is the following result that was pointed out to the author by Mark Teply. The left (resp. right) annihilator of an ideal $I$ is denoted by $l(I)$ (resp. $r(I)$ ).

Proposition 4.10. A semilocal $\operatorname{ring} R$ with $l(r(\operatorname{Jac}(R)))=\operatorname{Jac}(R)$ is a left Kasch ring.

Proof. Assume that $l(r(\operatorname{Jac}(R)))=\operatorname{Jac}(R)$ holds and that ${ }_{R} E$ be a simple module with $E \simeq(R a+\operatorname{Jac}(R)) / \operatorname{Jac}(R) \simeq R a /(R a \cap \operatorname{Jac}(R))$ for an $a \in R$. Since $a \notin \operatorname{Jac}(R)$, there exists by hypothesis $b \in r(\operatorname{Jac}(R))=\operatorname{Soc}\left({ }_{R} R\right)$ with $a b \neq 0$. Thus we have an isomorphism $\phi: E \rightarrow R a b$ with $\phi(r \bar{a}):=r a b$. Hence $R$ cogenerates all simple left $R$-modules; $R$ is a left Kasch ring. The converse is true for every left Kasch ring.

REMARK 4.11. Summarizing we have that $\tau_{c G}$ splits for the following clases of rings:

- semilocal left Kasch rings;

- left Kasch rings with finite right Goldie dimension;

- left Kasch, right CS-rings;

- left V-rings;

- local rings;

- prime left Goldie rings, not left primitive;

- products of integral domains;

- commutative semiperfect rings;

- commutative noetherian semilocal rings;

- any finite direct product of the rings above. 
Note that commutative noetherian rings are "stable rings; i.e. every hereditary torsion theory is stable. By Theorem 4.6 we know that $\tau_{c G}$ splits for semilocal stable rings.

REMARK 4.12. There are (local) rings with $\tau_{c G}$ splitting and additional reasonable good properties (like 'injective cogenerator' or 'artinian') that are still not QF. For instance F.Dischinger and W.Müller give a famous example of a local left PF-ring that is not right PF (see DM ), and Björks gives an example of a local two-sided artinian ring with $\operatorname{Jac}(R)^{2}=0$ that is not left self-injective (see $[\mathbf{B}]$ ).

On the other hand, even a two-sided artinian condition need not imply the splitting of $\tau_{c G}$. Let $k$ be a field and $R:=\left(\begin{array}{cc}k & k \\ 0 & k\end{array}\right)$. Then $R$ is two-sided artinian. The left simple $R$-module $R / \operatorname{Soc}\left({ }_{R} R\right)$ is injective but not projective, and hence by Theorem $4.6 \tau_{c G}$ does not split.

Proposition 4.13. Let $R$ be a left artinian ring with $\operatorname{Soc}\left(R_{R}\right) \subseteq \operatorname{Soc}\left({ }_{R} R\right)$. Then $\tau_{c G}$ splits.

Proof. Any left singular $R$-module $M$ is a $R / \operatorname{Soc}\left({ }_{R} R\right)$-module and so by hypothesis is also a $R / \operatorname{Soc}\left(R_{R}\right)$-module. By [R82, Theorem 3] $M$ is a left small $R$-module; by Theorem 4.6(h) $\tau_{c G}$ splits.

We would like to end this note with a remark: In view of Theorem 4.4, is there an example of a ring such that $\tau_{c G}$ is splitting but not cohereditary?

\section{References}

[BKN] L. Bican, T. Kepka, P. Nemec, Rings, modules and preradicals, Marcel Dekker, 1982

[B] J.-E. Björk, Rings satisfying certain chain conditions, J. Reine Angew. Math. 245, 63-73, 1970

[DM] F. Dischinger, W. Müller, Left PF is not right PF., Comm. Algebra 14(7), 1223-1227, 1986

[FM] C.Faith, P.Menal A counter example to Johns Conjecture, Proc. Amer. Math. Soc. 116, 21-26, 1992

[Ge] A.I. Generalov, A radical dual to Goldie torsion., Abelevy Grupy Moduli 11-12, 70-77, 1994

[G] J.S. Golan, Torsion theories, Pitman Monographs and Surveys in Pure and Appl. Math. 29. Longman, New York 1986

[GPY] J.L. Gómez Pardo, M.F. Yousif, Semiperfect min-CS rings, preprint 1998

$[\mathrm{H}] \quad$ M.Harada, On small submodules in the total quotient ring of a commutative ring, Rev. Union Mat. Argentina 28, 99-102, 1977

[HS] A.Harmancı, P.F.Smith, Relative injectivity and module classes, Comm. Alg. 20(9), 24712501,1992

[L] W.W. Leonard, Small modules, Proc. Amer. Math. Soc. 17, 527-531, 1966

[Lo] C.Lomp, On dual Goldie dimension, Diplomarbeit (MSc-Thesis), HHU Düsseldorf, Germany, 1996

[O] K. Oshiro, On Harada rings I, Math. J. Okayama Univ. 31, 161-178, 1989

[Oz] A.C. Özcan, Atik Moduller Yardimiyla Bazi Halkalarin Karakterizasyonlari ve Modullerin Parcalanislari ( Characterizations of some rings by small modules and decomposability of modules.), $\mathrm{PhD}$ thesis, Hacettepe University, Turkey, 1999

$[\mathrm{OH}]$ A.C. Özcan, A. Harmancı, Characterization of some rings by functor $Z^{*}()$, Turkish J. Math., 21(3), 325 - 331, 1997

[P] B.Pareigis, Radikale und kleine Moduln (Radicals and small modules), Bayer. Akad. Wiss., Math.-naturw. Kl., Sitzungsber. 1965, 185-199, 1966.

[R] V.S. Ramamurthi, The smallest left exact radical containing the Jacobson radical., Ann. Soc. Sci. Bruxelles Ser. I 96(4), 201- 206, 1982 
[R82] M. Rayar, On small and cosmall modules, Acta Math. Hungar, 39(4), 389-392, 1982

[R87] M. Rayar, A note on small rings, Acta Math. Hungar. 49(3-4), 381-383, 1987

[W91] R.Wisbauer, Foundations of Module and Ring Theory, Gordon and Breach, Reading, 1991

[W96] R.Wisbauer, Modules and algebras: bimodule structure and group actions on algebras, Longman, Harlow, 1996

Departamento de Matemática Pura, Universidade do Porto, 4050 Porto, Portugal

E-mail address: clomp@fc.up.pt 